\title{
Genetic Kidney Cancer Syndromes
}

Thai H. Ho, MD, PhD, and Eric Jonasch, $\mathrm{MD}^{\mathrm{b}}$

\begin{abstract}
Hereditary forms of renal cell carcinoma (RCC) have yielded clues regarding the molecular pathogenesis of sporadic RCC. The discovery of germline mutations in chromatin-modulating enzymes also defined a new hereditary RCC syndrome. Although histologically distinct RCC subtypes exist, emerging themes shared between hereditary and sporadic RCC include dysregulation of the von Hippel-Lindau tumor suppressor protein/hypoxia inducible factor axis, defective ciliogenesis, and aberrant tumor metabolism. This article describes the most common hereditary RCC syndromes and associated extrarenal manifestations. Recent evidence supports developing screening guidelines for early-onset RCC to identify persons with germline mutations in the absence of secondary clinical manifestations. ( $N$ Natl Compr Canc Netw 2014;12:1347-1355)
\end{abstract}

Hereditary renal cell carcinoma (RCC) has been estimated to account for $5 \%$ to $8 \%$ of all RCC cases, and extrarenal manifestations may present as early as 3 years of age (Table 1) ${ }^{1,2} \mathrm{RCC}$ is a diverse set of cancers that originate from the renal parenchyma. Histologic classifications include clear cell, papillary, chromophobe, and translocation; rare subtypes include renal medullary and collecting duct. Hereditary familial RCC syndromes have yielded clues regarding the molecular pathogenesis of sporadic RCC and have served as a framework for the development of targeted therapies. With diverse presen-

From the aDivision of Hematology and Oncology, Mayo Clinic, Scottsdale, Arizona, and bepartment of Genitourinary Medical Oncology, The University of Texas MD Anderson Cancer Center, Houston, Texas.

Submitted April 8, 2014; accepted for publication August 2, 2014. The authors have disclosed that they have no financial interests, arrangements, affiliations, or commercial interests with the manufacturers of any products discussed in this article or their competitors.

Correspondence: Eric Jonasch, MD, Department of Genitourinary Medical Oncology, The University of Texas MD Anderson Cancer Center, 1515 Holcombe Boulevard, Unit 1374, Houston, TX 77030. E-mail: ejonasch@mdanderson.org tations and incomplete penetrance of RCC, establishing screening guidelines for detecting early-onset RCC may help identify persons with germline mutations who have an increased risk of developing RCC.

\section{von Hippel-Lindau Disease}

von Hippel-Lindau (VHL) disease is an autosomal dominant disorder caused by germline mutations in the VHL gene, a tumor suppressor found on chromosome $3 \mathrm{p} 25 .{ }^{3}$ Patients inherit a nonfunctional VHL allele from a parent, or have a de novo germline VHL mutation. ${ }^{4} \mathrm{~A}$ stochastic secondary inactivation of the other allele leads to the development of renal cysts and tumors. The most common sporadic RCC histologic type (75\%) is clear cell RCC (ccRCC), which is also associated with loss of VHL function. Mechanisms of biallelic VHL inactivation in sporadic ccRCC include loss of heterozygosity at the VHL locus, somatic VHL mutations, and VHL hypermethylation. ${ }^{5}$ The VHL gene product, pVHL, acts as an oxygen sensor that regulates degradation of the hypoxia-inducible factors (HIFs) (Figure 1). The HIF family contains 3 subunits: HIF- $1 \alpha$, HIF- $2 \alpha$, and HIF$3 \alpha$. HIFs bind hypoxia-related elements to transactivate target genes, such as vascular endothelial growth factor (VEGF), involved in cellular adaptation to hypoxia. $\mathrm{pVHL}$ acts as the substrate recognition component of the ubiquitin E3 ligase complex. ${ }^{6}$ Recognition by $\mathrm{pVHL}$ requires the hydroxylation of HIF- $1 \alpha$ and HIF- $2 \alpha$ by an oxygen-dependent prolyl hydroxylase, and in normoxia, pVHL targets HIF-1 $\alpha$ and HIF- $2 \alpha$ for proteasomal degradation. ${ }^{7}$ Conversely, in hypoxic conditions or in pVHL-deficient cells, HIF- $1 \alpha$ and HIF- $2 \alpha$ bind to HIF$1 \beta$, forming a transcription factor leading to expression of hypoxia-responsive genes. ${ }^{8}$ In the setting of $\mathrm{pVHL}$ loss, inhibition of HIF- $2 \alpha$ is sufficient to suppress tumor formation. ${ }^{9}$ pVHL loss is also associated with Aurora 


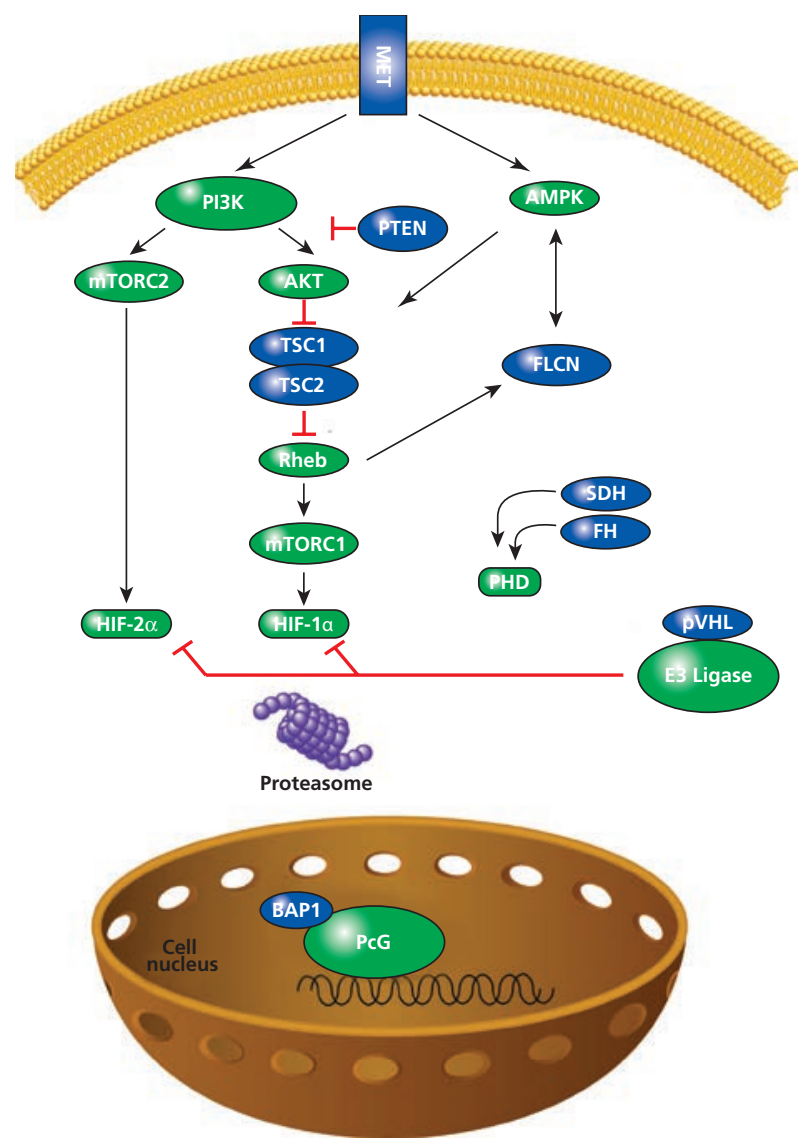

Figure 1 Genes mutated in hereditary kidney cancer syndromes. Mutations (blue) in MET, PTEN, TSC1/TSC2, FLCN, VHL, SDH, $F H$, and $B A P 1$ are linked to sporadic RCC and hereditary kidney cancer syndromes. In hereditary RCC syndromes, the age of onset is approximately 27 years younger than observed in the general population with sporadic RCC. TSC1/TSC2, VHL, and FLCN mutations also share features with ciliopathies, with cyst formation in target organs. $F H$ or $S D H$ mutations cause accumulation of tricarboxylic acid cycle intermediates that inhibit PHDs and also stabilize HIF-1 $\alpha$ and HIF- $2 \alpha$. Similarly, VHL mutations disrupt $\mathrm{pVHL}$ binding to HIF- $1 \alpha$ and HIF- $2 \alpha$, and HIF- $1 \alpha$ and HIF- $2 \alpha$ escape ubiquitin mediated proteolysis. Abbreviations: AMPK, adenosine monophosphate-dependent protein kinase; HIF, hypoxia-inducible factor; mTORC1, mammalian target of rapamycin complex 1; mTORC2, mTOR complex 2; PcG, polycomb group; PHD, prolyl hydroxylase; PI3K, phosphoinositide 3-kinase; RCC, renal cell carcinoma.

Adapted from Linehan WM, Bratslavsky G, Pinto PA, et al. Molecular diagnosis and therapy of kidney cancer. Ann Rev Med 2010;61:329-343.

kinase A overexpression and formation of visceral cysts, a common feature shared with other ciliopathies. ${ }^{10}$

VHL disease is characterized by an increased risk of kidney cysts, ccRCC, pheochromocytomas, and central nervous system hemangioblastomas (Table 1 and Figure 2).4,11 The mean age of RCC onset is 35 years, and RCC is the leading cause of death in persons affected by VHL disease. ${ }^{12}$ In a trial evaluating the use of sunitinib in VHL-associated lesions, responses were noted in renal lesions but not hemangioblastomas. ${ }^{13}$ Activation of the fibroblast growth factor (FGF) pathway may mediate escape from VEGF inhibition and a trial of dovitinib, an inhibitor of VEGF and FGF signaling, is underway in VHL (ClinicalTrials.gov identifier: NCT01266070), but results are not yet available. Other potential therapeutic interventions include Aurora kinase A inhibitors to restore primary cilia formation, but whether restoring cilia would reduce tumorigenesis in renal cyst mice models is unknown.

\section{Tuberous Sclerosis Complex}

Tuberous sclerosis complex is caused by germline mutations in the TSC1/2 genes, located on chromosomes $9 \mathrm{q} 34$ and $16 \mathrm{p} 13$, respectively. ${ }^{14}$ The mTOR complex 1 (mTORC1) signaling cascade is regulated by a heterodimer of TSC1 (hamartin) and TSC2 (tuberin), which function as tumor suppressors (Figure 1). Inactivation of TSC1/2 results in a derepression of mTORC1 inhibition. ${ }^{15}$ The mTOR pathway responds to both external and internal stimuli to regulate a diverse set of processes, including cell proliferation, nutrient abundance, protein synthesis, and cytoskeletal rearrangements.

Persons who inherit TSC1/2 mutations may develop hamartomas, angiomyolipomas, pulmonary lymphangioleiomyomatosis, subependymal giant cell astrocytomas, and RCC (Figure 3). ${ }^{16}$ Similar to those with VHL disease, persons with TSC1/2 mutations have kidney cysts that are associated with ciliary dysfunction. Angiomyolipomas cause morbidity through spontaneous hemorrhage, invasion of adjacent renal parenchyma, and chronic kidney disease. The identification of mTORC1 activation caused by TSC1/2 mutations led to clinical trials using sirolimus, an mTOR inhibitor (ClinicalTrials.gov identifier: NCT00457808), in patients with tuberous sclerosis complex. ${ }^{15}$ Everolimus was approved by the FDA for angiomyolipoma associated with tuberous sclerosis complex or sporadic lymphangioleiomyomatosis after a phase III trial showed a response rate of $42 \%$, defined as a reduction in angiomyolipoma volume of $50 \% .{ }^{17}$ In the study, $78 \%$ of the patients had bilateral angiomyolipomas and $40 \%$ had prior invasive procedures, suggesting that everolimus is 


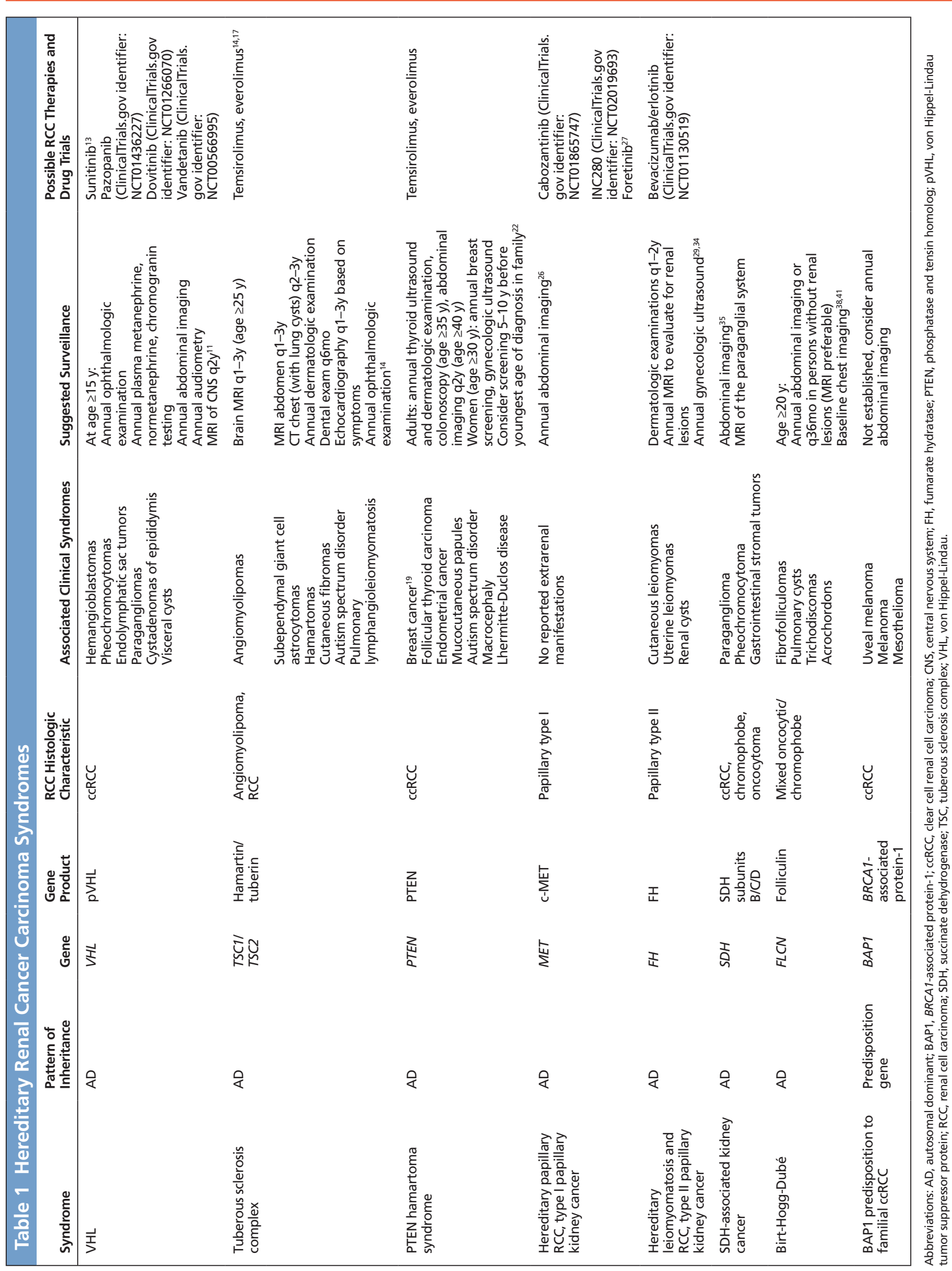


Ho and Jonasch

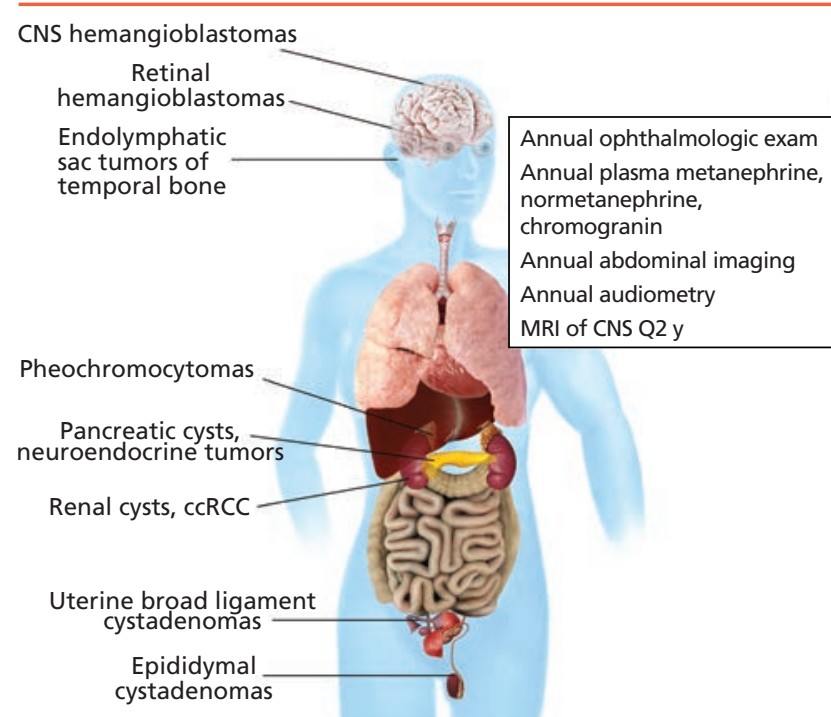

Figure 2 Clinical manifestations of von Hippel-Lindau (VHL) disease. VHL disease is an autosomal dominant hereditary cancer disorder in which affected persons either inherit a nonfunctional $V H L$ allele or have a de novo $\mathrm{VHL}$ mutation. Affected persons are at increased risk for tumor development in the CNS, pancreas, adrenal gland, kidney, and reproductive system.

Abbreviations: ccRCC, clear cell renal cell carcinoma; CNS, central nervous system.

an alternative for patients with multifocal disease or those ineligible for surgical intervention. Multiple renal cysts and angiomyolipomas develop, and renal disease is the leading cause of death in persons affected by tuberous sclerosis complex. ${ }^{18}$ Other causes of death include pulmonary lymphangioleiomyomatosis and subependymal giant cell astrocytomas.

\section{Phosphatase and Tensin Homolog Hamartoma Syndrome}

Phosphatase and tensin homolog (PTEN) hamartoma tumor syndrome is linked to inactivating germline mutations in PTEN, located on chromosome 10q23. ${ }^{19}$ Loss of PTEN function leads to a potent derepression of the phosphoinositide 3-kinase/AKT pathway, which stimulates cell growth and survival (Figure 1). ${ }^{20}$ PTEN mutations occur in approximately $5 \%$ of sporadic ccRCC cases, and a decrease in PTEN protein expression occurs in a significant number of sporadic RCCs. ${ }^{21}$

The lifetime risk of RCC in persons with germline PTEN mutations is estimated to be as high as $34 \%$, with typical onset of RCC at age 40 years. ${ }^{22}$ PTEN hamartoma tumor syndrome is also associated with a spectrum of clinical manifestations, in-

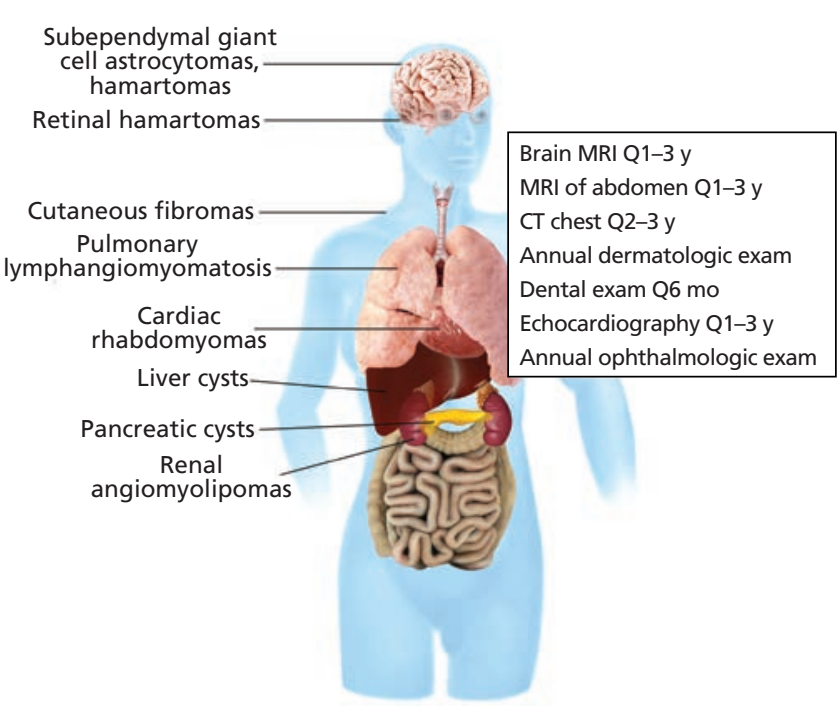

Figure 3 Clinical manifestations of tuberous sclerosis complex (TSC). TSC is an autosomal dominant hereditary cancer syndrome in which affected persons have germline inactivating mutations of TSC1/TSC2 genes. Affected persons are at increased risk for tumor development of the central nervous system, skin, heart, and kidney.

cluding breast cancer, endometrial cancer, follicular thyroid cancer, hamartomas, and mucocutaneous lesions (Figure 4). ${ }^{19}$ Although ascertainment bias may overestimate the risks, the age-related penetrance estimates reveal lifetime risks of $85 \%, 35 \%, 28 \%$, $9 \%$, and $6 \%$ for female breast cancer, thyroid cancer, endometrial cancer, colon cancer, and melanoma, respectively. ${ }^{22}$ Targeting the mTOR pathway in the context of PTEN deficiency has been proposed in PTEN hamartoma tumor syndrome-associated cancers, to exploit this potential vulnerability. A trial of sirolimus was completed in persons with PTEN hamartoma tumor syndrome (ClinicalTrials.gov identifier: NCT00971789), but the results are not available.

\section{Hereditary Papillary RCC, Type I Papillary Kidney Cancer}

Originally described as an inherited RCC that was histologically distinct from VHL disease, hereditary papillary RCC (HPRC) is an autosomal dominant cancer syndrome characterized by mutations in the proto-oncogene MET on chromosome 7q31 (Figure 1). ${ }^{23}$ Type I papillary RCCs occur in sporadic and hereditary forms, and somatic MET mutations are also found in approximately $10 \%$ of sporadic papillary kidney cancer. ${ }^{24} \mathrm{MET}$ encodes the cell surface receptor for hepatocyte growth factor that promotes 


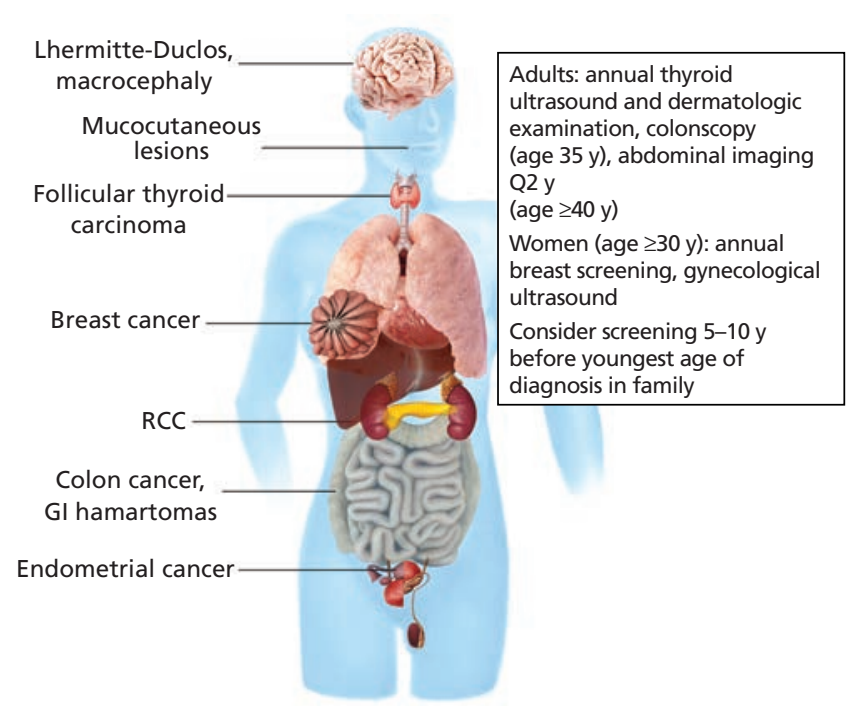

Figure 4 Clinical manifestations of phosphatase and tensin homolog hamartoma syndrome (PHTS). PHTS is an autosomal dominant hereditary cancer syndrome in which affected persons have germline inactivating mutations of PTEN. Affected persons are at increased risk for tumor development of the central nervous system, breast, colon, thyroid, skin, endometrium, and kidney.

Abbreviations: GI, gastrointestinal; RCC, renal cell carcinoma.

migration, invasion, proliferation, and angiogenesis. Unlike sporadic ccRCC and VHL disease, these RCC tumors lack $3 p$ alterations but exhibit trisomy 7, leading to multiple copies of MET and tumorigenesis initiated by the combination of a MET mutation and duplication of the mutated allele. ${ }^{25}$

Persons affected by this cancer syndrome can have bilateral involvement with more than 1000 tumors per kidney, suggesting the presence of microscopic precursors that may not be apparent on routine imaging. ${ }^{26}$ Extrarenal manifestations have not been reported. The presence of MET pathway activation or germline MET mutations was recently associated with responses to foretinib, a multikinase inhibitor targeting MET/VEGF receptor $2 .{ }^{27}$ A riskadapted approach of observing patients with small tumors $(<3 \mathrm{~cm})$, independent of location or number of tumors, was developed to guide surveillance based on a 10-year follow-up study of parenchymal-sparing surgery in hereditary RCC. ${ }^{28}$ Papillary RCC is hypoechoic and can be missed by ultrasound. ${ }^{29}$ Active surveillance with CT/MRI is recommended until a kidney tumor reaches $3 \mathrm{~cm}$ to mitigate the risk of metastatic disease and preserve renal function.

\section{Hereditary Leiomyomatosis and RCC, Type II Papillary Kidney Cancer}

Hereditary leiomyomatosis and RCC (HLRCC) is characterized by a germline mutation in $\mathrm{FH}$ (fumarate hydratase), found on chromosome $1 \mathrm{q} 42 .{ }^{30} \mathrm{FH}$ is a Krebs cycle enzyme that converts fumarate to malate, and FH inactivation causes a metabolic shift to aerobic glycolysis in $\mathrm{FH}$-deficient kidney cancer. ${ }^{31}$ The buildup of fumarate inhibits prolyl hydroxylase (PHD), interferes with the recognition of HIF-1 $\alpha$ and HIF- $2 \alpha$ by pVHL, and leads to an increase in VEGF-mediated angiogenesis (Figure 1). ${ }^{32}$

Affected persons can present with cutaneous leiomyomas, uterine leiomyomas, and type II papillary RCC (Figure 5). ${ }^{29,30}$ Systemic therapy specific for patients with HLRCC has yet to be fully developed. Expression profiling of an Fh1-deficient mouse model and HLRCC cell lines shows a common pathway of dysregulated glycolytic genes, suggesting that FH deficiency may sensitize cells to glycolytic inhibitors. ${ }^{31}$ For example, the glycolytic inhibitor 2-deoxy-Dglucose was used to treat a patient with HLRCC after experiencing disease progression while receiving an mTOR inhibitor, and although the patient experienced hypoglycemia consistent with inhibition of glycolysis, no antitumor effect occurred. ${ }^{33}$ The median age of onset for the development of papillary RCC is 37 years, and individuals in approximately $15 \%$ of families with HLRCC develop disease. ${ }^{1,29}$ Although a $3-\mathrm{cm}$ threshold is used to guide management of RCC in other hereditary syndromes, immediate surgical management of any identified HLRCC-associated renal tumors is suggested because of aggressive growth and a propensity for early metastases. ${ }^{34}$ Papillary RCC is hypoechoic, can be missed by ultrasound, and is best screened with CT/MRI imaging. ${ }^{29}$ Uterine leiomyomas also develop earlier in women affected by cutaneous leiomyomas compared with the general population. ${ }^{34}$ Because of symptomatic leiomyomas, women affected by HLRCC have higher rates of hysterectomy and undergo hysterectomy at a younger age, indicating a need for family planning counseling.

\section{Succinate Dehydrogenase-Associated Kidney Cancer}

Succinate dehydrogenase (SDH)-associated kidney cancer is linked to a germline mutation in genes that 
Ho and Jonasch

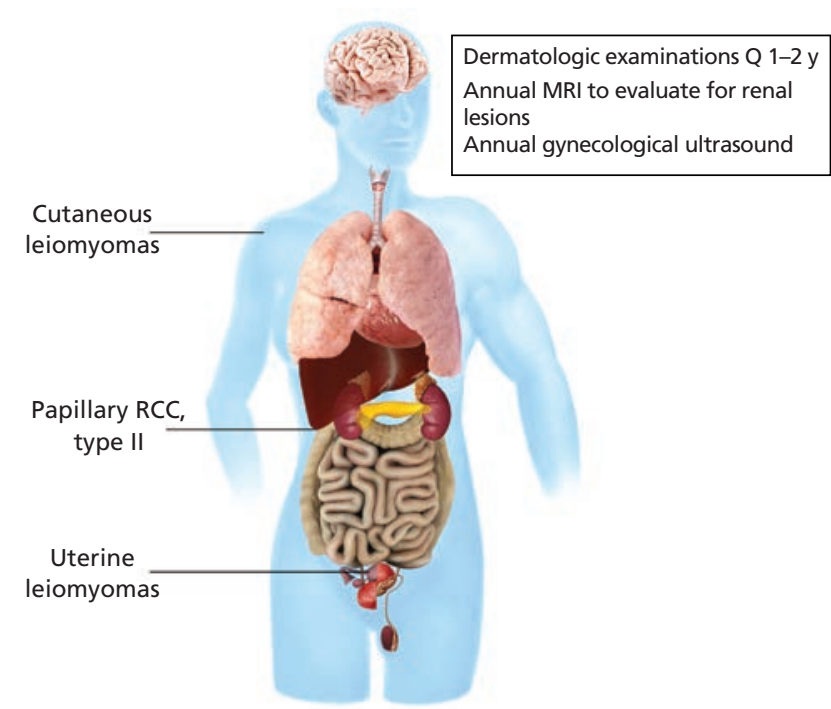

Figure 5 Clinical manifestations of hereditary leiomyomatosis and renal cell carcinoma (HLRCC). HLRCC is an autosomal dominant hereditary cancer syndrome in which affected persons have germline inactivating mutations of $F H$. Affected persons are at increased risk for tumor development of skin, uterus, and kidney.

Abbreviation: RCC, renal cell carcinoma.

encode for subunits of the tricarboxylic acid cycle enzyme, SDH. ${ }^{35} \mathrm{SDH}$ genes include SDHA (chromosome 5p15), SDHB (chromosome 1p36), SDHC (chromosome 1q23), and SDHD (chromosome 11q23). Mutations in SDH cause accumulation of succinate and, similar to fumarate in HLRCC, also inhibit prolyl hydroxylation of HIF- $1 \alpha$ and HIF- $2 \alpha$ (Figure 1). ${ }^{36}$

RCC may be the only clinical presentation in persons with germline SDHB, SDHC, or SDHD mutations. ${ }^{2}$ Extrarenal manifestations include pheochromocytomas, paragangliomas, carotid body tumors, and gastrointestinal stromal tumors. Because of the rarity of these tumors, no established screening guidelines have been developed. Mutations in Krebs cycle enzymes shift the cells toward increased glucose uptake, aerobic glycolysis, and fatty acid synthesis. ${ }^{37}$ The increased dependence on glycolytic pathways suggests that inhibitors of glucose uptake, glycolysis, and fatty acid synthesis could exploit the vulnerability of these tumors. Among the SDH subunit mutations, SDHB and SDHD carriers are more likely to develop RCC, with a median age of onset of 30 years. ${ }^{1}$ Symptoms related to pheochromocytomas and paragangliomas may present as early as 3 years of age in germline carriers, supporting a role for germline testing of SDHB and SDHD at initial presenta- tion. ${ }^{2}$ Subsequent surveillance for renal masses and extrarenal manifestations is likely warranted in this population. ${ }^{35}$

\section{Birt-Hogg-Dubé Syndrome}

Birt-Hogg-Dubé (BHD) is an autosomal dominant syndrome caused by mutations of the FLCN gene, located on chromosome $17 \mathrm{p} 11 .{ }^{38}$ The FLCN gene encodes for folliculin, a downstream target of both adenosine monophosphate-dependent protein $\mathrm{ki}$ nase and mTORC1 signaling; folliculin also localizes to cilia (Figure 1). ${ }^{39}$ Loss of folliculin function leads to mTORC1 activation and dysregulation of ciliogenesis.

Clinically, BHD is characterized by fibrofolliculomas, pulmonary cysts, and early-onset RCC, commonly with mixed oncocytoma and chromophobe histologic characteristics. ${ }^{38}$ Similar to the VHL and tuberous sclerosis complex disease syndromes, the BHD-associated pulmonary cysts share characteristics consistent with a ciliopathy. The risk of developing RCC at age 70 years has been estimated to be $16 \%$ in patients with $\mathrm{BHD}$, with characteristic hybrid histologic characteristics of chromophobe RCC and oncocytomas. ${ }^{40}$ Similar to VHL disease and HPRC, active surveillance is recommended for the management of renal lesions less than $3 \mathrm{~cm} .{ }^{28,41}$ When adjusted for age, persons with BHD have a 50 -fold increased risk of pneumothorax. ${ }^{42}$ Pulmonary consultation is recommended for those at risk of ambient atmospheric pressure changes, and smoking cessation may reduce morbidity associated with pulmonary cysts. mTOR inhibitors have prolonged survival of folliculin-deficient mice and have been proposed to treat the clinical manifestations of BHD. 43 However, a trial of topical rapamycin to treat BHDassociated fibrofolliculomas did not reduce the size or number of cutaneous lesions. ${ }^{44}$

\section{BRCA1-Associated Protein-1 Predisposition to Familial ccRCC}

Systematic sequencing of sporadic ccRCC identified loss-of-function mutations in histone-modulating enzymes, such as the BRCA1-associated protein-1 (BAP1) (5\%-15\%) gene on chromosome 3p21.1..$^{21,45}$ BAP1 functions as a nuclear deubiquitinase that interacts with polycomb group $(\mathrm{PcG})$ proteins at open 
chromatin and promotes double-strand break repair (Figure 1). ${ }^{46}$

After the discovery of BAP1 mutations in sporadic ccRCC, germline mutations were linked with familial ccRCC syndromes. ${ }^{47}$ Germline BAP1 mutations are also associated with uveal melanomas and mesotheliomas. However, the BAP1 syndrome is rare, making it difficult to assess its prevalence in the general population and estimate tumor risks to guide surveillance. The use of general guidelines for earlyonset kidney screening may be considered based on age of initial presentation. ${ }^{1}$

\section{Overlapping Mechanisms Between Hereditary and Sporadic ccRCC}

The overlap of gene mutations in VHL, TSC1, MTOR, PTEN, and BAP1 in both hereditary and sporadic ccRCC has increased the understanding of tumorigenesis. The 4 most frequently mutated genes

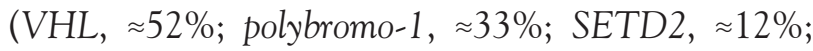
and BAP1, $\approx 10 \%$ ) are located on chromosome $3 \mathrm{p}$, a region that is deleted in more than $90 \%$ of sporadic ccRCCs, supporting a mechanism of biallelic inactivation of these tumor suppressors. ${ }^{21}$ In parallel with the clinical benefit seen with mTOR inhibitors in the tuberous sclerosis complex syndrome, a subset of patients with sporadic ccRCC and TSC1 inactivation or MTOR mutations may experience a durable response to $\mathrm{mTOR}$ inhibitors. ${ }^{48}$ PTEN $(\approx 4 \%)$ and
PIK3CA $(\approx 3 \%)$ mutations occur in approximately $4 \%$ of sporadic ccRCC cases, supporting a phenotypic convergence on the PI3K/mTOR axis (Figure 1). An integrated analysis of molecular pathways identified a common metabolic phenotype of increased glycolysis between sporadic ccRCC and HLRCC. ${ }^{21}$ Krebs cycle mutations in HLRCC and SDH-associated kidney cancer stabilize HIF and shift metabolism to increased reliance on aerobic glycolysis, characterized as "the Warburg effect," by mobilizing nutrients (nucleotides, amino acids, lipids) for cell proliferation. ${ }^{32}$ Similarly, in sporadic ccRCC, biallelic inactivation of VHL leads to HIF stabilization, and tumors with metabolic shifts toward aerobic glycolysis are associated with a worse survival. ${ }^{21}$

\section{Establishing a Role for Early-Onset Kidney Cancer Screening}

The median age of patients with sporadic RCC was 64 years in an analysis of the SEER 17 registry program, which was considerably older than the median age of 37 years in a cohort of patients with hereditary kidney cancer. At the NCI Urologic Oncology Branch, patients with VHL, BHD, HLRCC, HPRC, and SDHB syndromes had median ages of onset ranging from 35 to 50 years. Because the median age of presentation for hereditary RCC is 27 years younger than that for RCC observed in the general population, patients with RCC who are 46 years old or younger should consider ge-

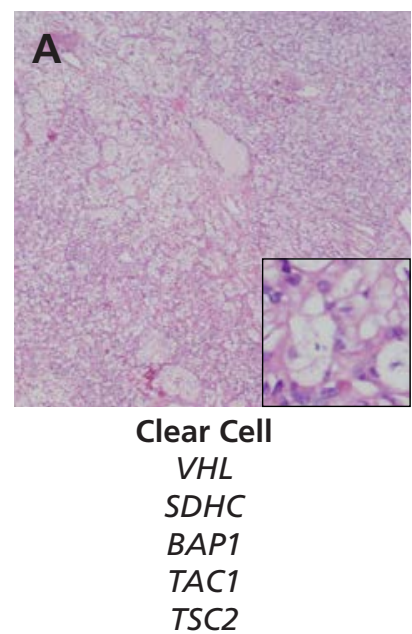

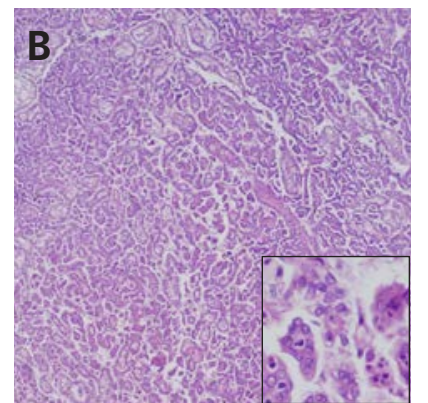

Papillary,

Type I

MET

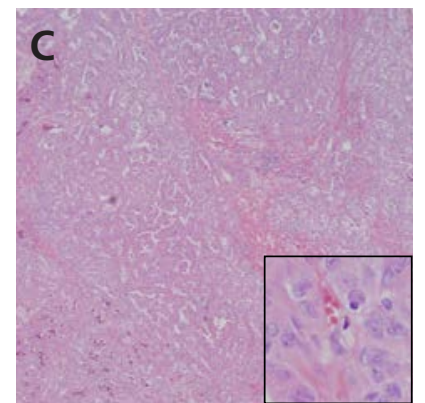

Papillary,

Type II

$\mathrm{FH}$

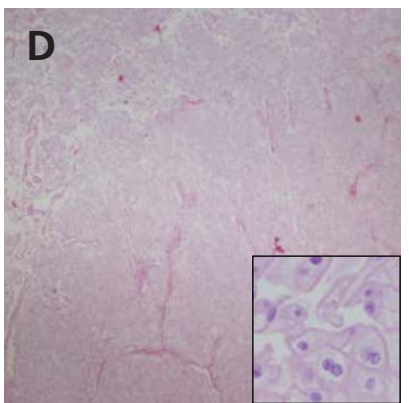

Chromophobe

FLCN

TSC1

TSC2

PTEN

Figure 6 Germline testing of selected genes based on renal cell carcinoma (RCC) histology: (A) clear cell, (B) papillary type I, (C) papillary type II, and (D) chromophobe. Persons with RCC aged 46 years or younger should be considered for genetic counseling and germline mutations testing, even in the absence of secondary clinical manifestations. The RCC histologic characteristics may guide which germline mutations to test. (Hematoxylin and eosin stain; original magnification x10 and x40 [inset]) Courtesy of Dr. Melissa L. Stanton, Mayo Clinic Arizona. 
Ho and Jonasch

netic counseling and germline mutation testing even in the absence of secondary clinical manifestations. ${ }^{1}$ The histology of early-onset RCC may guide which germline mutations to test (Figure 6). ${ }^{49}$

\section{Conclusions}

Patients with hereditary RCC syndromes provide the clinician with unique diagnostic, surveillance, and therapeutic challenges. Many of the described syndromes demonstrate highly distinct phenotypes, allowing clinical findings to guide genetic testing. Once a hereditary RCC syndrome is identified, careful adherence to surveillance strategies, appropriate management of the renal and extrarenal disease manifestations, and attention to at-risk family members will lead to improvement in patient outcome.

The hereditary drivers of several nonsyndromic RCC cohorts have long eluded investigators. Advances in genomewide sequencing technologies have led to the identification of additional mutations in RCC and to the discovery of the most recently described BAP1 familial RCC syndrome. ${ }^{47}$

Despite the complexity of networks involved in hereditary and sporadic RCC, these alterations share a common dysregulation of the HIF-VEGF axis and aberrant tumor metabolism. ${ }^{21}$ The identification of genes linked to RCC syndromes has led to the development of clinical trials selecting for patients affected by hereditary syndromes (Table 1). ${ }^{21}$ Additional drug targets may exist outside the mTOR and VEGF pathways, such as proteins that influence metabolic sensitivity or chromatin remodeling proteins. Ultimately, the identification of new druggable targets for sequential or combination therapies may provide a more robust form of therapy for patients who have sporadic and hereditary RCC.

The observed early onset of hereditary RCC indicates that primary preventative strategies are more likely to increase life expectancy in affected persons than administering targeted therapies in the metastatic setting. Persons affected with RCC at age 46 years or younger should be considered for genetic counseling and germline mutational testing, even in the absence of secondary clinical manifestations. ${ }^{1}$ As life expectancy increases, additional clinical manifestations in hereditary RCC syndromes will undoubtedly become apparent, and clinicians and families must maintain vigilant and report new findings.

\section{References}

1. Shuch B, Vourganti S, Ricketts CJ, et al. Defining early-onset kidney cancer: implications for germline and somatic mutation testing and clinical management. J Clin Oncol 2013.

2. Ricketts CJ, Forman JR, Rattenberry E, et al. Tumor risks and genotypephenotype-proteotype analysis in 358 patients with germline mutations in SDHB and SDHD. Hum Mutat 2010;31:41-51.

3. Latif F, Tory K, Gnarra J, et al. Identification of the von Hippel-Lindau disease tumor suppressor gene. Science 1993;260:1317-1320.

4. Prowse $\mathrm{AH}$, Webster $\mathrm{AR}$, Richards FM, et al. Somatic inactivation of the VHL gene in Von Hippel-Lindau disease tumors. Am J Hum Genet 1997;60:765771.

5. Latif F, Duh FM, Gnarra J, et al. von Hippel-Lindau syndrome: cloning and identification of the plasma membrane $\mathrm{Ca}(++)$-transporting ATPase isoform 2 gene that resides in the von Hippel-Lindau gene region. Cancer Res 1993;53:861867.

6. Cockman ME, Masson N, Mole DR, et al. Hypoxia inducible factor-alpha binding and ubiquitylation by the von Hippel-Lindau tumor suppressor protein. J Biol Chem 2000;275:25733-25741

7. Maxwell PH, Wiesener MS, Chang GW, et al. The tumour suppressor protein VHL targets hypoxia-inducible factors for oxygen-dependent proteolysis. Nature 1999;399:271-275.

8. Semenza GL. Targeting HIF-1 for cancer therapy. Nat Rev Cancer 2003;3:721732.

9. Kondo K, Kim WY, Lechpammer M, Kaelin WG Jr. Inhibition of HIF2alpha is sufficient to suppress pVHL-defective tumor growth. PLoS Biol 2003;1:E83.

10. $\mathrm{Xu} \mathrm{J}$, Li H, Wang B, et al. VHL inactivation induces HEF1 and Aurora kinase A. J Am Soc Nephrol 2010;21:2041-2046.

11. Binderup ML, Bisgaard ML, Harbud V, et al. Von Hippel-Lindau Disease (vHL). National Clinical Guideline for Diagnosis and Surveillance in Denmark. 3rd ed. Dan Med J 2013;60:B4763.

12. Maher ER, Neumann HP, Richard S. von Hippel-Lindau disease: a clinical and scientific review. Eur J Hum Genet 2011;19:617-623.

13. Jonasch E, McCutcheon IE, Waguespack SG, et al. Pilot trial of sunitinib therapy in patients with von Hippel-Lindau disease. Ann Oncol 2011;22:26612666.

14. Krueger DA, Northrup H. Tuberous sclerosis complex surveillance and management: recommendations of the 2012 International Tuberous Sclerosis Complex Consensus Conference. Pediatr Neurol 2013;49:255-265.

15. Laplante M, Sabatini DM. mTOR signaling in growth control and disease. Cell 2012;149:274-293.

16. Crino PB, Nathanson KL, Henske EP. The tuberous sclerosis complex. N Engl J Med 2006;355:1345-1356.

17. Bissler JJ, Kingswood JC, Radzikowska E, et al. Everolimus for angiomyolipoma associated with tuberous sclerosis complex or sporadic lymphangioleiomyomatosis (EXIST-2): a multicentre, randomised, doubleblind, placebo-controlled trial. Lancet 2013;381:817-824.

18. Shepherd CW, Gomez MR, Lie JT, Crowson CS. Causes of death in patients with tuberous sclerosis. Mayo Clin Proc 1991;66:792-796.

19. Pilarski R, Burt R, Kohlman W, et al. Cowden syndrome and the PTEN hamartoma tumor syndrome: systematic review and revised diagnostic criteria. J Natl Cancer Inst 2013;105:1607-1616.

20. Sun H, Lesche R, Li DM, et al. PTEN modulates cell cycle progression and cell survival by regulating phosphatidylinositol 3,4,5,-trisphosphate and Akt/ protein kinase B signaling pathway. Proc Natl Acad Sci U S A 1999;96:61996204.

21. Comprehensive molecular characterization of clear cell renal cell carcinoma. Nature 2013;499:43-49.

22. Tan MH, Mester JL, Ngeow J, et al. Lifetime cancer risks in individuals with germline PTEN mutations. Clin Cancer Res 2012;18:400-407.

23. Schmidt L, Duh FM, Chen F, et al. Germline and somatic mutations in the tyrosine kinase domain of the MET proto-oncogene in papillary renal carcinomas. Nat Genet 1997;16:68-73.

24. Linehan WM, Srinivasan R, Schmidt LS. The genetic basis of kidney cancer: a metabolic disease. Nat Rev Urol 2010;7:277-285. 
Genetic Kidney Cancer Syndromes

25. Zhuang Z, Park WS, Pack S, et al. Trisomy 7-harbouring non-random duplication of the mutant MET allele in hereditary papillary renal carcinomas. Nat Genet 1998;20:66-69.

26. Ornstein DK, Lubensky IA, Venzon D, et al. Prevalence of microscopic tumors in normal appearing renal parenchyma of patients with hereditary papillary renal cancer. J Urol 2000;163:431-433.

27. Choueiri TK, Vaishampayan U, Rosenberg JE, et al. Phase II and biomarker study of the dual MET/VEGFR2 inhibitor foretinib in patients with papillary renal cell carcinoma. J Clin Oncol 2013;31:181-186.

28. Herring JC, Enquist EG, Chernoff A, et al. Parenchymal sparing surgery in patients with hereditary renal cell carcinoma: 10-year experience. J Urol 2001;165:777-781.

29. Toro JR, Nickerson ML, Wei MH, et al. Mutations in the fumarate hydratase gene cause hereditary leiomyomatosis and renal cell cancer in families in North America. Am J Hum Genet 2003;73:95-106.

30. Tomlinson IP, Alam NA, Rowan AJ, et al. Germline mutations in FH predispose to dominantly inherited uterine fibroids, skin leiomyomata and papillary renal cell cancer. Nat Genet 2002;30:406-410.

31. Ashrafian $\mathrm{H}$, O'Flaherty L, Adam J, et al. Expression profiling in progressive stages of fumarate-hydratase deficiency: the contribution of metabolic changes to tumorigenesis. Cancer Res 2010;70:9153-9165.

32. Pollard PJ, Spencer-Dene B, Shukla D, et al. Targeted inactivation of fh1 causes proliferative renal cyst development and activation of the hypoxia pathway. Cancer Cell 2007;11:311-319.

33. Yamasaki T, Tran TA, Oz OK, et al. Exploring a glycolytic inhibitor for the treatment of an FH-deficient type-2 papillary RCC. Nat Rev Urol 2011;8:165171.

34. Smit DL, Mensenkamp AR, Badeloe S, et al. Hereditary leiomyomatosis and renal cell cancer in families referred for fumarate hydratase germline mutation analysis. Clin Genet 2011;79:49-59.

35. Ricketts CJ, Shuch B, Vocke CD, et al. Succinate dehydrogenase kidney cancer: an aggressive example of the Warburg effect in cancer. J Urol 2012;188:20632071.

36. Selak MA, Armour SM, MacKenzie ED, et al. Succinate links TCA cycle dysfunction to oncogenesis by inhibiting HIF-alpha prolyl hydroxylase. Cancer Cell 2005;7:77-85.
37. Pollard PJ, Briere JJ, Alam NA, et al. Accumulation of Krebs cycle intermediates and over-expression of HIFlalpha in tumours which result from germline FH and SDH mutations. Hum Mol Genet 2005;14:2231-2239.

38. Menko FH, van Steensel MA, Giraud S, et al. Birt-Hogg-Dube syndrome: diagnosis and management. Lancet Oncol 2009;10:1199-1206.

39. Luijten MN, Basten SG, Claessens T, et al. Birt-Hogg-Dube syndrome is a novel ciliopathy. Hum Mol Genet 2013;22:4383-4397.

40. Houweling AC, Gijezen LM, Jonker MA, et al. Renal cancer and pneumothorax risk in Birt-Hogg-Dube syndrome; an analysis of 115 FLCN mutation carriers from 35 BHD families. Br J Cancer 2011;105:1912-1919.

41. Stamatakis L, Metwalli AR, Middelton LA, et al. Diagnosis and management of BHD-associated kidney cancer. Fam Cancer 2013;12:397-402.

42. Zbar B, Alvord WG, Glenn G, et al. Risk of renal and colonic neoplasms and spontaneous pneumothorax in the Birt-Hogg-Dube syndrome. Cancer Epidemiol Biomarkers Prev 2002;11:393-400.

43. Baba M, Furihata M, Hong SB, et al. Kidney-targeted Birt-Hogg-Dube gene inactivation in a mouse model: Erk1/2 and Akt-mTOR activation, cell hyperproliferation, and polycystic kidneys. J Natl Cancer Inst 2008;100:140_ 154.

44. Gijezen LM, Vernooij M, Martens $\mathrm{H}$, et al. Topical rapamycin as a treatment for fibrofolliculomas in Birt-Hogg-Dube syndrome: a double-blind placebocontrolled randomized split-face trial. PLoS One 2014;9:e99071.

45. Pena-Llopis S, Vega-Rubin-de-Celis S, Liao A, et al. BAP1 loss defines a new class of renal cell carcinoma. Nat Genet 2012;44:751-759.

46. Yu H, Pak H, Hammond-Martel I, et al. Tumor suppressor and deubiquitinase BAP1 promotes DNA double-strand break repair. Proc Natl Acad Sci U S A 2014;111:285-290.

47. Popova $T$, Hebert L, Jacquemin V, et al. Germline BAP1 mutations predispose to renal cell carcinomas. Am J Hum Genet 2013;92:974-980.

48. Voss MH, Hakimi AA, Pham CG, et al. Tumor genetic analyses of patients with metastatic renal cell carcinoma and extended benefit from mTOR inhibitor therapy. Clin Cancer Res 2014;20:1955-1964.

49. Linehan WM. Evaluation and screening for hereditary renal cell cancers. Can Urol Assoc J 2013;7:324-325. 